\title{
Autonomous Internal Control System for Small to Medium Firms
}

\author{
Juan M. Corchado ${ }^{1}$, M. Lourdes Borrajo ${ }^{2}$, and J. Carlos Yáñez ${ }^{3}$ \\ ${ }^{1}$ Departamento de Informática y Automática, \\ University of Salamanca, Plaza de la Merced s/n, 37008 Salamanca, Spain \\ corchado@usal.es \\ ${ }^{2}$ Department of Computer Science, \\ University of Vigo, Campus As Lagoas, s/n, 32004 Ourense, Spain \\ lborrajo@uvigo.es \\ ${ }^{3}$ Department of Financial Accounting, \\ University of Vigo, Campus as Lagoas, s/n, 32004 Ourense, Spain \\ jcyanez@uvigo.es
}

\begin{abstract}
Small to medium enterprises require an internal control mechanism in order to monitor their modus operandi and to analyse whether they are achieving their goals. A tool for the decision support process has been developed based on a case-based reasoning system that automates the internal control process. The objective of the system is to facilitate the process of internal auditing. The system analyses the data that characterises each one of the activities carried out by the firm, then determines the state of each activity, calculates the associated risk, detects the erroneous processes, and generates recommendations to improve these processes. The developed model is composed of two case-based reasoning systems. One is used to identify the activities that may be improved and the other to determine how the activities could be improved. Each of the two subsystems uses a different problem solving method in each of the steps of the reasoning cycle. The system has been tested in 22 small and medium companies in the textile sector, located in the northwest of Spain during the last 39 months and the results obtained have been very encouraging.
\end{abstract}

\section{Introduction}

Small to medium enterprises require an internal control mechanism in order to monitor their modus operandi and to analyse whether they are achieving their goals. Such mechanisms are constructed around series of organizational policies and specific procedures dedicated to giving reasonable guarantees to their executive bodies. This group of policies and procedures are named "controls", and they all conform to the structure of internal control of the company. As a consequence of this, the need for periodic internal audits has arisen. Nevertheless the evaluation and the prediction of the evolution of these types of systems, characterized by their great dynamism, are, in general, complicated. It is necessary to construct models that facilitate the analysis of work carried out in changing environments, such as finance. 
The processes carried out inside a firm are grouped in functional areas [7] denominated "Functions". A Function is a group of coordinated and related activities, which are necessary to reach the objectives of the firm and are carried out in a systematic and iterative way [14]. The functions that are usually carried out within a firm are: Purchases, Cash Management, Sales, Information Technology, Fixed Assets Management, Compliance to Legal Norms and Human Resources. In turn, each one of these functions is broken down into a series of activities. For example, the function Information Technology is divided in the following activities: Computer Plan Development, Study of Systems, Installation of Systems, Treatment of Information Flows and Security Management.

Each activity is composed of a number of tasks. For example, the activity Computer Plan Development, belonging to the function Information Technology, can be divided in the following tasks:

1. Definition of the required investment in technology in the short and medium time.

2. Coordination of the technology investment plan and the development plan of the company.

3. Periodic evaluation of the established priorities on the technology investment plan to identify their relevance.

4. Definition of a working group focused in the identification and control of the information technology policy.

5. Definition of a communication protocol, in both directions: bottom-up and topdown, to involve the firm employees in the maintenance strategic plan.

Control procedures have also to be established in the tasks to ensure that the established objectives are achieved.

The developed system is composed of two fundamental subsystems [5]:

- Subsystem ISA (Identification of the State of the Activity) whose objectives are: to identify the state or situation of each one of activities of the company and to calculate the risk associated with this state.

- Subsystem GR (Generation of Recommendations), whose goal is to generate recommendations to reduce the number of inconsistent processes in the firm.

Both subsystems are implemented using a case-based reasoning (CBR) system [1,

$12,17,13]$. The CBR system associated with each subsystem uses different problem solving techniques and shares the same case memory $[11,15]$.

The rest of this article is structured as follows: first, the proposed CBR based model is presented. Then, its results are evaluated. Finally the conclusions are presented.

\section{Neuro-symbolic System for Internal Control}

This section describes the internal control system in detail. Although the aim is to develop a generic model useful in any type of small to medium enterprise, the initial work has focused in the textile sector to facilitate the research and its evaluation. The model here presented may be extended or adapted for other sectors. Twenty two companies from the North-west of Spain have collaborated in this research, some of them manufacture products for major firms such as Inditex and others work mainly 
for the Spanish market. The companies have different levels of automation and all of them were very interested in a tool such as the one developed in the framework of this investigation. After analyzing the data relative to the activities developed within a given firm, the constructed system is able to determine the state of each of the activities and calculate the associated risk. It also detects any erroneous processes and generates recommendations for improving these processes. As shown below the problem solving mechanism developed takes its decision using the help of a couple of CBR systems whose memory has been fed with cases constructed with information provided by the firm and with prototypical cases identified by 34 internal control experts who have collaborated and supervised the model developed.

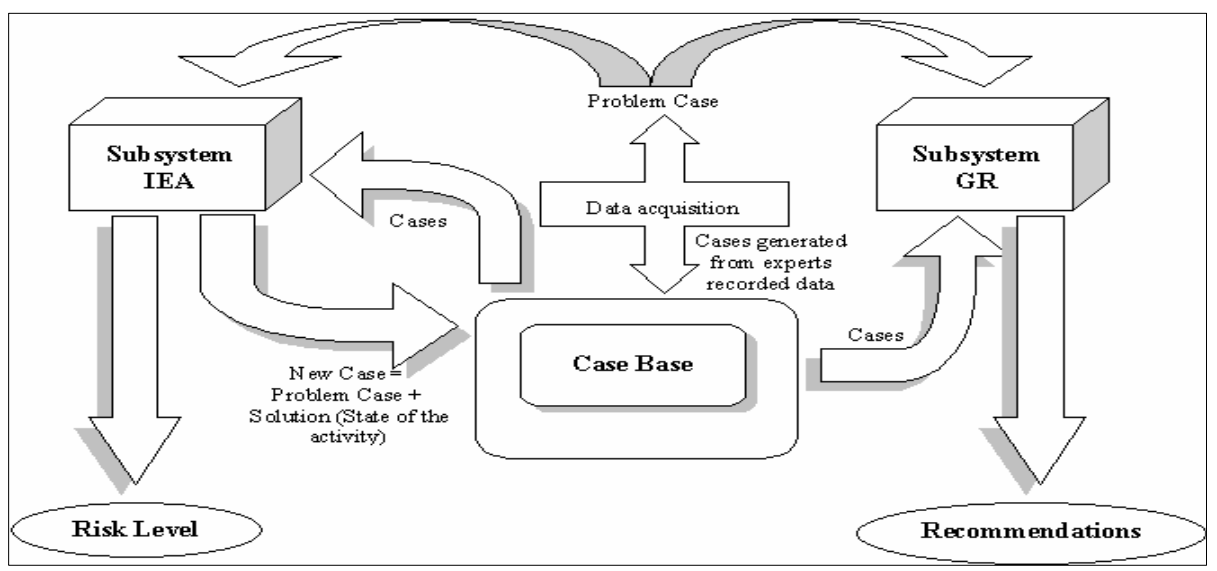

Fig. 1. System reasoning process

The cycle of operations of the developed case based reasoning system is based on the classic life cycle of a CBR system $[1,18]$. This cycle is executed twice, since the system bases its operation on two CBR subsystems (subsystem ISA-Identification of the State of the Activity and subsystem GR-Generation of Recommendations), as can be seen in Figure 1. Both subsystems share the same case base (Table 1 shows the attributes of a case) and a case represents the "shape" of a given activity developed in the company.

Every time that it is necessary to obtain a new estimate of the state of an activity, the hybrid system evolves through several phases. This evolution allows the system, on the one hand, (i) to identify the latest situations most similar to the current situation, (ii) to adapt the current knowledge to generate an initial estimate of the risk of the activity being analysed, and on the other hand, (iii) to identify old situations that serve as a basis to detect the erroneous processes developed within the activity and (iv) to select the best of all possible activities. The activity selected will then serve as guide for the establishment of a set of recommendations that allow the activity, its function and the company itself to develop in a more positive way. The retain phase guarantees that the system evolves in parallel with the firm, basing the corrective actions on the calculation of the error made previously. The following sections describe the different phases of the proposed model. 


\subsection{Data Acquisition}

The data used to construct the model were obtained from a set of surveys:

1. External auditors' surveys. Through the results of the surveys, each one of the functions and activities of a firm is assigned a level importance. This information allows the system to calculate the control risk associated with an activity. Also, it allows the system to prioritise the recommendations for improving the business processes. This data is stored in the system database.

2. Experts' surveys on the different functional areas. The second type of survey was carried out among several experts in the different functional areas of various firms. This type of survey attempts to reflect the experience of the experts in their different fields. For each activity, the survey presents two possible situations: the first one tries to reflect the situation of an activity with an incorrect activity state and the second one tries to reflect the situation of an activity with a satisfactory activity state. Both situations will be valued by the expert using a percentage. The data acquired by the surveys have been used to build the prototype cases for the initial case base.

Table 1 shows the case structure that constitutes the case base.

Table 1. Case structure.

\begin{tabular}{|c|c|c|c|c|c|c|}
\hline \multicolumn{6}{|c|}{ PROBLEM } & SOLUTION \\
\hline $\begin{array}{c}\text { Case } \\
\text { number }\end{array}$ & $\begin{array}{l}\text { Input } \\
\text { vector }\end{array}$ & $\begin{array}{c}\text { Function } \\
\text { number }\end{array}$ & $\begin{array}{l}\text { Activity } \\
\text { number }\end{array}$ & Reliability & $\begin{array}{c}\text { Degree of } \\
\text { membership }\end{array}$ & $\begin{array}{l}\text { Activity } \\
\text { State }\end{array}$ \\
\hline
\end{tabular}

Each case is composed of the following attributes:

- Case number: Unique identification: positive integer number.

- Input vector: Information about the tasks ( $\mathrm{n}$ sub-vectors) that constitute an industrial activity: $\left(\left(\mathrm{IR}_{1}, \mathrm{~V}_{1}\right),\left(\mathrm{IR}_{2}, \mathrm{~V}_{2}\right), \ldots,\left(\mathrm{IR}_{\mathrm{n}}, \mathrm{V}_{\mathrm{n}}\right)\right)$ for $\mathrm{n}$ tasks. Each task sub-vector has the following structure $\left(\mathrm{GI}_{\mathrm{i}}, \mathrm{V}_{\mathrm{i}}\right)$ :

- $\quad \mathrm{IR}_{\mathrm{i}}$ : importance rate for this task within the activity. It can only take one of the following values: VHI (Very high importance), HI (High Importance), AI (Average Importance), LI (Low Importance), VLI (Very low importance)

- $\quad \mathrm{V}_{\mathrm{i}}$ : Value of the realization state of a given task: a positive integer number (between 1 and 10).

- Function number: Unique identification number for each function

- Activity number: Unique identification number for each activity

- Reliability: Percentage of probability of success. It represents the percentage of success obtained using the case as a reference to generate recommendations.

- Degree of membership: $\left(\left(\mathrm{n}_{1}, \mu_{1}\right),\left(\mathrm{n}_{2}, \mu_{2}\right), \ldots,\left(\mathrm{n}_{\mathrm{k}}, \mu_{\mathrm{k}}\right)\right)$

- $\quad n_{\mathrm{i}}$ : represents the $\mathrm{i}^{\text {st }}$ cluster

- $\quad \mu_{\mathrm{i}}$ : represents the membership value of the case to the cluster $n_{i}$

- Activity State: degree of perfection of the development of the activity, expressed by percentage. 


\subsection{Subsystem ISA (Identification of the State of the Activity)}

The subsystem ISA (Identification of the State of the Activity) identifies the state or situation of each of the firm's activities and calculates the risk associated with this situation. The system uses the data for the activity, introduced by the firm's internal auditor, to construct the problem case. For each task making up the activity analyzed, the problem case is composed of the value of the realization state for that task, and its level of importance within the activity (according to the internal auditor).

In this way, a problem case for an activity of $n$ tasks, will be composed of a vector such as: $\left(\left(\mathrm{IR}_{1}, \mathrm{~V}_{1}\right),\left(\mathrm{IR}_{2}, \mathrm{~V}_{2}\right), \ldots,\left(\mathrm{IR}_{\mathrm{n}}, \mathrm{V}_{\mathrm{n}}\right)\right)$ where:

- $\mathrm{IR}_{\mathrm{i}}$ : importance rate for this task within the activity. It can only take one of the following values: VHI (Very high importance), HI (High Importance), AI (Average Importance), LI (Low Importance), VLI (Very low importance).

- $\mathrm{V}_{\mathrm{i}}$ : Value of the realization state of a given task. It is a positive integer number (between 1 and 10).

\subsubsection{Retrieval Phase}

This phase has as its objective the retrieval of $\mathrm{K}$ cases - the most similar cases to the problem case. This is carried out by means of a technique of cluster-based similarity.

Using the fuzzy C-means method [3,4], the most similar cases belonging to the case base are grouped together. With this clustering method, $n$ clusters are obtained, each of them containing cases exclusively from the case base. Each case will have associated a degree of membership for each cluster, expressed by a percentage. A representative case or centre is identified for each cluster.

The object of the following step is to identify the cluster containing the cases nearest to the problem case in order to reduce the size of the problem space. Thus, the problem case is compared with the centre of each cluster. The cluster selected will be the one whose centre is located closest to the problem case. The distance between the problem case and the cluster centres was calculated using the Mahalanobis:

$$
d_{M}\left(x_{i}, x_{j}\right)=\left(x_{i}-x_{j}\right) \sum^{-1}\left(x_{i}-x_{j}\right)^{T}
$$

Since a precise estimate, provided by the system, is necessary, only those cases with a high degree of membership to the cluster and a high reliability will be retrieved. The reliability indicates the percentage of probability of success using this case in the process of generating recommendations. In our system, only those $\mathrm{K}$ cases with a degree of membership to the cluster greater than $65 \%$ and with a reliability higher than $50 \%$ will be retrieved. These two percentages have been established by the auditors interviewed.

Figure 2 shows the pseudocode for this retrieve phase. $X$ represents the set of cases that introduces the knowledge about an activity, $v_{p}$ represents the vector of characteristics (attributes) that describes a new problem, n_cluster represents the amount of clusters that the system is looking forward to obtaining (in this case $n \_$cluster $=3$ has been chosen), max_iter represents the highest number of iterations of the algorithm and it has been initialized at 100, $P$ represents the set of centres of the $n_{-}$cluster clusters, $U$ is the matrix of memberships, $u_{m i}$ represents the degree of 
membership of the case $i$ to the cluster $m$ whose center is $c_{m}$ and $K$ is the set of retrieved cases.

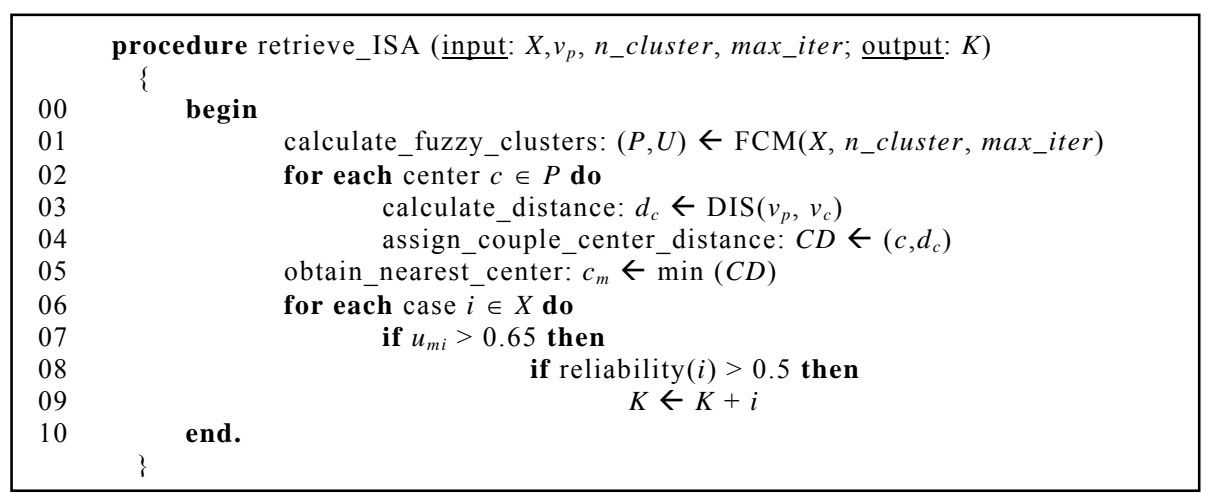

Fig. 2. Pseudocode of the retrieve phase of the subsystem ISA (Identification of the State of the Activity)

Fuzzy clustering techniques are used because of the size of the database and the need to group the most similar cases together in order to help retrieve the cases that most resemble the given problem.

Fuzzy clustering techniques are especially interesting for non-linear or ill-defined problems, making it possible to treat tasks involved in the processing of massive quantities of redundant or imprecise information. It allows the available data to be grouped into clusters with fuzzy boundaries, expressing uncertain knowledge.

\subsubsection{Re-use Phase}

This phase aims to obtain an initial estimation of the state of the activity analysed. In order to obtain this estimation, RBF networks are used $[9,6,8]$. As in the previous stage, the number of attributes of the problem case depends on the activity analyzed. Therefore it is necessary to establish an RBF network system, one for each of the activities to be analysed.

When the new problem case is presented, the cluster whose centre is closest to the case is identified and a set of $K$ cases is retrieved from the system. These cases are used by the RBF network as a training group that allows it to adapt its configuration to the new problem encountered before generating the initial estimation.

The topology of each of the RBF networks used in this task consists of: an input layer with as many neurons as attributes possessed by the input vector which constitutes the problem descriptor $\left(\left(\mathrm{IR}_{1}, \mathrm{~V}_{1}\right),\left(\mathrm{IR}_{2}, \mathrm{~V}_{2}\right), \ldots,\left(\mathrm{IR}_{\mathrm{n}}, \mathrm{V}_{\mathrm{n}}\right)\right)$, a hidden layer with 50 centres, and an output layer with a single neuron corresponding to the variable to be estimated (correction level or state of activity analysed in x percent).

Figure 3 shows the pseudocode of the algorithm that roughly illustrates the steps that need to be followed in order to obtain an initial estimate, using the $K$ cases retrieved in the previous phase and the descriptor of the problem for which an estimate needs to be made. In the algorithm, $v_{p}$ represents the vector of characteristics (attributes) that form the problem case, $K$ is the group of most relevant retrieved 
cases, confRBF is the group of neurons that make up the topology of the RBF network and $s_{i}$ represents the initial solution generated for the current problem.

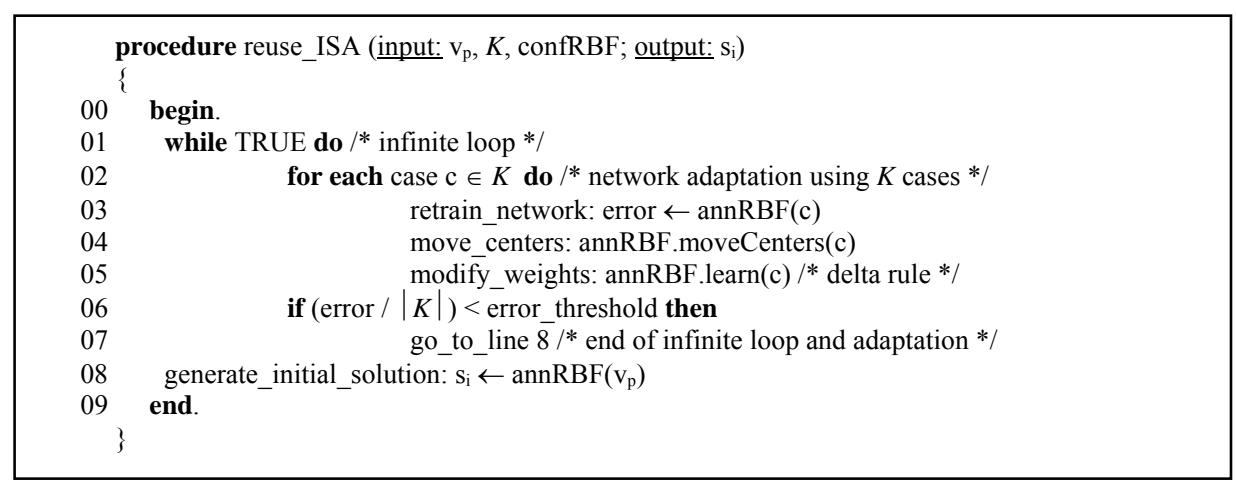

Fig. 3. Pseudocode of the reuse phase of the ISA (Identification of the State of the Activity) subsystem.

The RBF network is characterized by its ability to adapt, to learn rapidly, and to generalize (especially in interpolation tasks). Specifically, within this system the network acts as a mechanism capable of absorbing knowledge about a certain number of cases and generalizing from them. During this process, the RBF network, interpolates and carries out predictions without forgetting part of those already carried out. The system's memory acts as a permanent memory capable of maintaining many cases or experiences while the RBF network acts as a short term memory, able to recognize recently learnt patterns and to generalize from them.

\subsubsection{Revision Phase}

The objective of the revision phase is to confirm or refute the initial solution proposed by the RBF network, thereby obtaining a final solution and calculating the control risk.

In view of the initial estimation or solution generated by the RBF network, the internal auditor will be responsible for deciding if the solution is accepted. For this it is based on the knowledge he/she retains, specifically, knowledge about the company with which he/she is working. If it considers that the estimation given is valid, the system will take the solution as the final solution and in the following phase of the CBR cycle, a new case will be stored in the case base consisting of the problem case and the final solution. The system will assign the case an initial reliability of $100 \%$. If on the other hand, the internal auditor considers the solution given by the system to be invalid, he will give his own solution which the system will take as the final solution and which together with the problem case will form the new case to be stored in the case base in the following phase. This new case will be given a reliability of $30 \%$. This value has been defined taking into account the opinion of various auditors in terms of the weighting that should be assigned to the personal opinion of the internal auditor.

From the final solution: state of activity, the system calculates the control risk associated with the activity. Every activity developed in the business sector has a risk 
associated with it that indicates the negative influence that affects the good operation of the firm. In other words, the control risk of an activity measures the impact that the current state of the activity has on the business process as a whole. In this study, the level of risk is valued at three levels: low, medium and high. The calculation of the level of control risk associated with an activity, is based on the current state of the activity and its level of importance. This latter value was obtained after analysing data obtained from a series of questionnaires (98 in total) carried out by auditors throughout Spain. In these questionnaires the auditors were asked to rate subjects from 1-10 according to the importance or weighting of each activity in terms of the function that it belonged to. The higher the importance of the activity, the greater its weighting within the internal control system.

The level of control risk was then calculated from the level of importance given to the activity by the auditors and the final solution obtained after the revision phase. For this purpose, if-then rules are employed.

\subsubsection{Retention Phase}

The last phase of the ISA (Identification of the State of the Activity) subsystem is the incorporation of the system's memory of what has been learnt after resolving a new problem. Once the revision phase has been completed, after obtaining the final solution, a new case (problem + solution) is constructed, which is stored in the system's memory. Apart from the overall knowledge update involving the insertion of a new case within the system memory, the hybrid system presented carries out a local adaptation of the knowledge structures that it uses.

The fuzzy cluster system contained within the prototypes related to the activity corresponding to the new case are reorganised in order to respond to the appearance of this new case, modifying their internal structure and adapting themselves to the new knowledge available.

In this way, the RBF network uses the new case to carry out a complete learning cycle, updating the position of its centres and modifying the value of the weightings that connect the hidden layer with the output layer.

The learning process is continuous whereby the RBF acts as a mechanism capable of absorbing knowledge of a certain number of cases, and to use them as a base with which to generalise. During this process the RBF network interpolates and makes predictions without forgetting part of predictions that have already been made. The system's memory acts as a permanent memory capable of maintaining many cases or experiences while the RBF network acts as a short term memory, capable of recognising recently learnt patterns and generalising on them.

\subsection{GR Subsystem (Generation of Recommendations)}

The objective of this subsystem is to carry out recommendations to help the internal auditor decide which actions to take, once the previous stages of the subsystem have concluded, in order to improve the company's internal and external processes. This subsystem is totally dependent on the previous subsystem since part of the case (problem+solution) is generated in the ISA - Identification of the State of Activity Subsystem (see Figure 1). 


\subsubsection{Retrieval Phase}

The second subsystem (GR-Generation of Recommendations) is used to generate recommendations that can guide the internal auditor in his task of deciding the actions to be taken in order to improve the state of the activity analysed. In order to recommend changes in the execution of the business processes it is necessary to compare the current situation in an activity, represented by the problem case + solution, generated by the ISA (Identification of the State of the Activity) Subsystem, with those cases from the case base which best reflect the business management.

To this end, only cases most similar to the problem case are worked on. Given that the cluster whose centre was closest to the case problem was identified during the recuperation phase of the Identification of the State of Activity, the cases of this cluster will be used in the next adaptation phase. The process followed in this retrieval phase is based on the use of query relaxation [10] so that initially the cases retrieved from the case base meet the following conditions:

1. The solution or state of activity must be $15-20 \%$ superior to the final solution generated by the previous subsystem. If enough cases are not retrieved (25 is considered to be enough) the percentages are relaxed further, increasing in range by $5 \%$.

2. Furthermore, they should possess a level of pertinence to the higher cluster of $75 \%$ and a level of reliability of over $50 \%$. These two constant values have been established by the auditors.

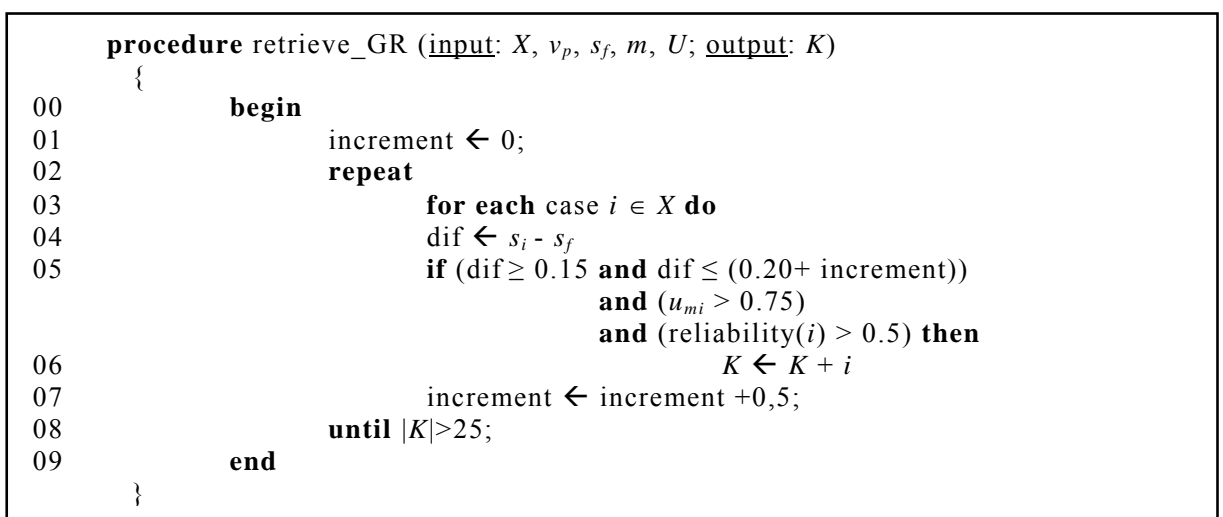

Fig. 4. Pseudocode of the retrieval phase in the Generation of Recommendations subsystem.

Figure 4 shows the retrieval process adopted, where $\mathrm{X}$ stands for the case group which represents the knowledge of a determined activity that exists within the memory of the system, $v_{p}$ represents the vector of attributes that describes the problem case, $s_{f}$ is the final solution generated in the ESA subsystem as a solution to the problem case, $s_{i}$ is the solution to case $i, m$ is the identifier of the cluster whose centre is closest to the problem case, $U$ is the matrix of pertinences, $u_{m i}$ is the level of pertinence of case $i$ to cluster $m$ and $K$ is the most relevant case group retrieved. 


\subsubsection{Reuse Phase}

Given that the objective of this subsystem is to generate a series of recommendations from the problem case, it is necessary to search for a case from the case base (or a combination of various cases) which serve as a guide to generate recommendations, comparing this/these case/s with the problem case. This comparison will allow the company to detect which processes need to be modified - in other words, which tasks need to be improved.

As already explained above, in the retrieval phase, the cases obtained are those which best reflect the most favourable overall activity state presented by the activity analysed. From all these cases, in this phase of adaptation, the subsystem should select the case which maximises the value of each of the tasks $\left(\mathrm{V}_{\mathrm{i}}\right)$ taking into account the level of importance $\left(\mathrm{IR}_{\mathrm{i}}\right)$ or weighting that each task has for the overall activity. In this way, the problem of selecting a case from all those retrieved can be made similar to a multi-criteria decision-making problem where the alternatives are the different cases retrieved and the objective is to maximise the values of these tasks (which will then represent the attributes).

In this study, the initial version of the Electre method $[2,16]$ has been used in order to tackle the problem of choosing one of the alternatives. The Electre method proposes a strategy for reducing the size of all the possible solutions, through the segregation of the most favourable case group from another group which encapsulates the least favourable cases. The application of such a method will produce the selection of one or various cases from among those retrieved.

The Electre method is based on the fact that the vector of preferential weightings subjectively associated with each attribute is known. As in this study, the weighting of an attribute (represented by its level of importance) is different for each alternative, and it is necessary to obtain a single weightings vector for the attributes of the group of alternatives or retrieved cases. In this case, to obtain the weighting vector is carried out by calculating for each attribute the median weightings for the attribute in question, for all the different alternatives.

On the other hand, as a solution, Electre returns the best alternative, or group of alternatives in the event that there is no single prevalent alternative. Given that for the generation of recommendations it is necessary to begin with a single alternative, where an output to a multicriteria decision method gives various alternatives, their combination will be used, taking the median value for each attribute.

Figure 5 shows the pseudocode for the reuse phase where $K$ is the group of most relevant cases retrieved in the previous phase, $v_{e l}$ is the case or alternative obtained after the adaptation phase from group of cases $K, v_{e l(j)}$ is the value of the attribute $j$ of the case $v_{e l}, C$ is the group of alternatives or cases obtained as output by the Electre method.

The case obtained as a result of the Electre method represents the objective to be reached for the activity analysed or the standard to be followed in order to meet the objectives of the company or, specifically, the objective associated with the activity. In this way, the recommendations which are generated retrospectively, will be used to ensure that the various tasks that make up the problem case form a situation which is as similar as possible to the case obtained at the exit of the Electre method. 


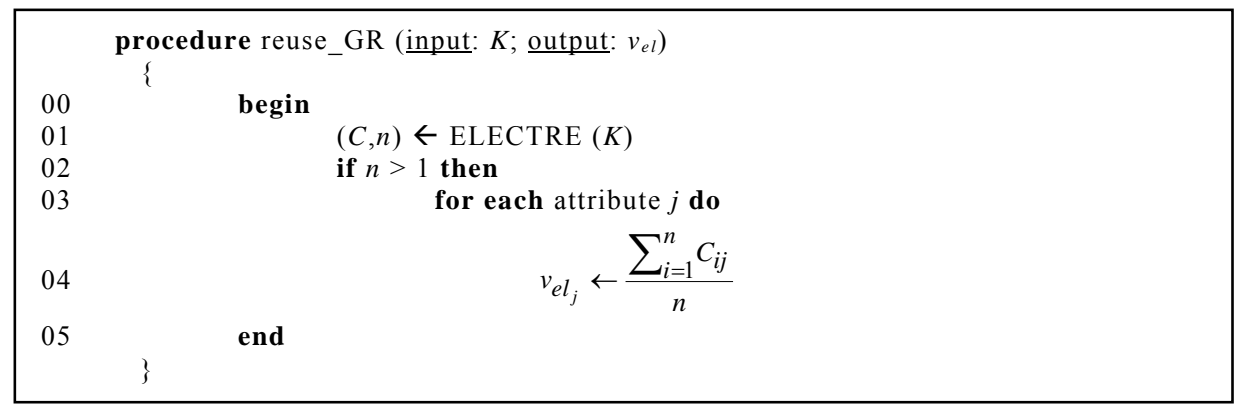

Fig. 5. Pseudocode of the reuse phase of the GR (Generation of Recommendations) subsystem.

In this way, in order to generate the recommendations, the output from the Electre method is compared to the problem case, comparing the values $\left(\mathrm{V}_{\mathrm{i}}\right)$ of each of the attributes or tasks in each case. The objective is to detect which tasks should be improved, establishing an order of priorities in terms of weighting $\left(\mathrm{IR}_{\mathrm{i}}\right)$ of each task over the overall weighting of the activity. In other words, to identify the possible deviations of the activity and to appreciate the extent of deviations in terms of the tasks' level of importance $\left(\mathrm{IR}_{\mathrm{i}}\right)$. In this way, the system generates recommendations related to the inconsistent processes found, that is, the differences between the values of the attributes in the problem case and those in the objective case (considered as the standard) obtained by the Electre method, representing the potential recommendations of the auditor.

The group of attributes of stored cases in the case base represent the overall values that both experts in each activity and the auditors have judged to be effective (from the surveys carried out) given the characteristics of the company. Since the characteristics of the current case (problem) are similar to the objective case obtained, the auditor can argue that the values of the attributes must also be similar. This provokes a more convincing argument than basing it on probabilities and estimated losses or risks.

The generation of control recommendations by comparing the values of the current case with those of past cases has also eliminated other problems such as the lack of outputs or pre-defined results. Many possible values exist as well as a large number of combinations that could be included in the recommendations of the auditor. But not all the combinations are valid; some combinations may not be feasible or make sense. In contrast to the CBRs, both the expert systems and the neuron networks will need to have possible outputs specified for them previously.

Based on the predictions and recommendations generated by the system, the internal auditor may inform the company of inconsistent processes and the measures that should be adopted to resolve them. This is a decision support system that facilitates the auditing process for internal auditors.

\subsubsection{Retain Phase}

After the time necessary for correcting the errors detected, the firm is evaluated again. Auditing experts consider that three months are enough to allow the evolution of the company towards a more favourable state. If it is verified that the erroneous processes 
and the level of risk have diminished, the retention phase is carried out, modifying the case used to generate the recommendations. The reliability (percentage of successful identifications) of this case is thereby increased by $10 \%$. In contrast, when the firm happens not to have evolved to a better state, the reliability of the case is decreased in $10 \%$. Furthermore, those cases whose level of reliability is smaller than $15 \%$ are eliminated, and the remaining cases are grouped into fuzzy clusters.

\section{Results}

The hybrid system developed has been tested over 29 months in 22 small to medium companies (12 medium-sized and 10 small) in the textile sector, located in the northwest of Spain. The data employed to generate prototype cases, in order to construct the system's case bases, have been obtained after performing 98 surveys with auditors from Spain, as well as 34 surveys from experts within different functional areas of the firms within the sector.

In order to test the system various complete operation cycles of the system were carried out. In other words, each of the companies' activities were evaluated, obtaining a level of risk and generating recommendations. These recommendations were communicated to the company's internal auditor and they were given a limit of three months to elaborate and apply an action plan based on the recommendations. The action plan's objective was to reduce the number of inconsistent processes within the company. After three months, a new analysis of the company was made and the results obtained were compared with those of the previous three months. This process was repeated every three months.

Results obtained demonstrate that the application of the recommendations generated by the system causes a positive evolution in firms. This evolution is reflected in the reduction of erroneous processes. Results obtained demonstrate that the application of the recommendations generated by the system causes a positive evolution in firms. The indicator used to determine the positive evolution of the companies was the state of each of the activities analysed. If, after analysing one of the company's activities, it is proven that the state of the activity (valued between 1 and 100) has increased over the state obtained in the previous three month period, it can be said that the erroneous processes have been reduced within the same activity. If this improvement is produced in the majority of activities (above all in those of most relevance within the company), the company has improved its situation.

In order to reflect as reliably as possible the ideal system for resolving the problem, the results from the analysis of the 22 companies were compared with those of 5 companies in which the recommendations generated by the system have not been applied. In these five companies, the activities were analysed from the beginning of the three month period until the end, using the ISA (Identification of the State of the Activity). The recommendations generated by the second subsystem were not presented to the firm managers (and consequently, the recommendations were not applied).

In order to analyse the results obtained, it is necessary to consider that a number of the recommendations implied costs that the companies were not able to permit or that 
supposed a long term installation. Therefore, companies are considered to have followed the recommendations if they followed at least $70 \%$. On the other hand, the evaluation process was ceased in two of the companies analysed at the request of the companies themselves. Specifically, only one year's data is available for one company while in the case of the other, only data from the first 21 months is available.

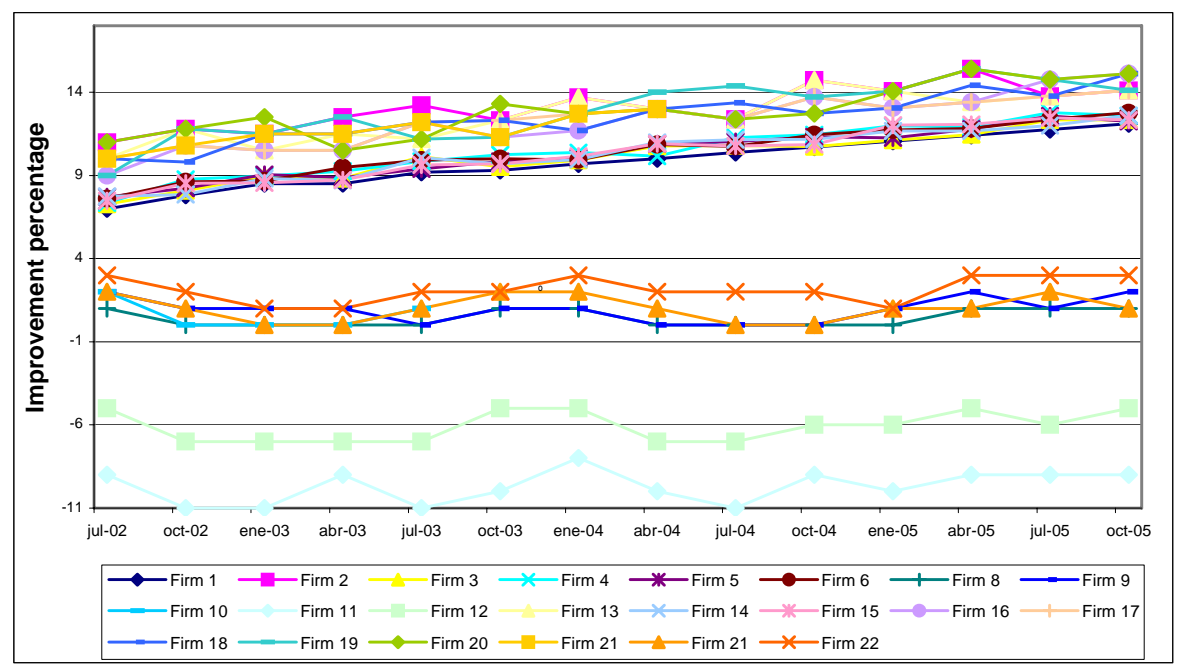

Fig. 6. Firms' evolution

The results obtained were as follows:

1. Of the 22 companies analysed, in those in which the recommendations generated by the system were applied, the results were (see Fig. 6):

a) In 15 companies, the number of inconsistent processes was reduced, improving the state of activities by an average of $11.5 \%$.

b) In 5 of these companies, no improvement was detected in the state of activities. In other words, the application of the recommendations generated by the system did not have any effect on the activities of the company. In two of these companies, the experiment had to be aborted after one year, and 21 months, respectively, because the companies themselves did not allow us to complete the analysis. In the other three companies (in which the system was applied for the full 39 month period), after studying the possible reasons for the results, it has been concluded that the recommendations given were not completely followed, with only certain measures applied and the majority of recommendations ignored.

c) In two companies the inconsistent processes increased, in other words the application of recommendations generated by the system, prejudiced the positive evolution of the company. Once the situation in each of the companies had been analysed, it was concluded that in both there was a high level of disorganisation, without a clearly defined set of objectives. This meant that any attempt to change the business organisation actually worsened the situation.

In general, it could be said that these results demonstrate the suitability of the techniques used for their integration in the developed intelligent control system. The 
best results occurred in the companies of smaller size. This is due to the fact that these firms have a greater facility to adapt and adopt the changes suggested by the system's recommendations.

2. On the other hand, for the 5 companies in which the recommendations generated by the system were not applied, the results were as follows: four of them improved their results, although reaching an average productivity that was $4 \%$ below the median of the other companies that did use the system. The fifth company analysed ceased operations before the end of the first year of evaluation.

\section{Conclusions}

This article presents a neuro-symbolic system that uses two CBR systems employed as a basis for hybridization of a multicriteria decision-making method, a fuzzy clustering method, and an RBF net. As such, the model developed combines the complementary properties of connectionist methods with the symbolic methods of Artificial Intelligence.

The used reasoning model can be applied in situations that satisfy the following conditions:

1. Each problem can be represented in the form of a vector of quantified values.

2. The case base should be representative of the total spectrum of the problem.

3. Cases must be updated periodically.

4. Enough cases should exist to train the net.

The prototype cases used for the construction of the case base are artificial and have been created from surveys carried out with auditors and experts in different functional areas. The system is able to estimate or identify the state of the activities of the firm and their associated risk. Furthermore the system generates recommendations that will guide the internal auditor in the elaboration of action plans to improve the processes in the firm.

Estimation in the environment of firms is difficult due to the complexity and the great dynamism of this environment. However, the developed model is able to estimate the state of the firm with precision, and propose solutions that make it possible to improve each state. The system will produce better results if it is fed with cases related to the sector in which it will be used. This is due to the dependence that exists between the processes in the firms and the sector where the company is located. Future experiments will help to identify how the constructed prototype will perform in other sectors and how it will have to be modified in order to improve its performance.

Although the defined model has not been tested in big firms, it is believed that it could work adequately, although changes would take place more slowly than in small and medium firms. Steps toward this direction have been taken and it is expected that an evaluation of the system will soon be possible in a major international company from the textile sector. 


\section{References}

1. Aamodt A. and Plaza E. (1994). Case-Based Reasoning: foundational Issues, Methodological Variations, and System Approaches. AICOM. Vol. 7. No 1, Marzo 1994.

2. Barba-Romero, S. y Pomeral, J. (1997). Decisiones Multicriterio. Fundamentos teóricos y utilización práctica. Colección de Economía. Servicio de publicaciones Universidad de Alcalá.

3. Bezdek J. C. (1981). Pattern Recognition with Fuzzy Objective Function Algorithms. Plenum Press, New York.

4. Bezdek, J.C., Keller, J.M., Krishnapuram, R. and Pal, N.R. (1999). Fuzzy Models and Algorithms for Pattern Recognition and Image Processing. Kluwer Academic Publishers, Norwell.

5. Borrajo, L.(2003). Sistema híbrido inteligente aplicado a la auditoría de los sistemas internos. Phd Thesis. Teses de doutoramento da Universidade de Vigo. Universidade de Vigo (Spain). ISBN: 84-8158-274-3. December, 2003.

6. Corchado, J.M., Díaz, F., Borrajo, L. and Fdez-Riverola F. (2000). Redes Neuronales Artificiales: Un enfoque práctico. Departamento de publicaciones de la Universidad de Vigo.

7. Corchado, J.M., Borrajo, L., Pellicer, M.A. and Yáñez, J.C. (2004). Neurosymbolic System for Business Internal Control. LNCS Volume 3275/2004. Springer-Verlag. ISSN:0302-9743.

8. Fdez-Riverola, F. and Corchado, J.M. (2004). FSfRT: Forecasting System for Red Tides. Applied Intelligence. Special Issue on Soft Computing in Case-Based Reasoning. ISSN 0924-669X. Vol 21, num 3, pp 251-264.

9. Fritzke, B. (1994). Fast Learning with Incremental RBF Networks. Neural Processing Letters. Vol. 1. No. 1. pp. 2-5.

10.Gardingen, D. \& Watson, I. (1998). A Web Based Case-Based Reasoning System for HVAC Sales Support. In, Applications \& Innovations in Expert Systems VI. Milne, R., Macintosh, A. \& Bramer, M. (Eds.), pp. 11- 23. Springer, London. ISBN 1-85233-087-2

11.Hunt, J. and Miles, R. (1994). Hybrid case-based reasoning. The Knowledge Engineering Review. Vol. 9:4. pp. 383-397.

12.Kolodner J. (1993). Case-Based Reasoning. San Mateo. CA, Morgan Kaufmann. 1993.

13.Lenz M., Bartsch-Spörl B., Burkhard D. and Wees S. (eds.) 1998. Case-based Reasoning Technology: From Fundations to Applications, Springer Verlag, LNAI 1400 .

14.Mas, J. and Ramió, C. (1997). La Auditoría Operativa en la Práctica. Ed. Marcombo, Barcelona.

15.Medsker L. R. (1995). Hybrid Intelligent Systems. Kluwer Academic Publishers.

16.Romero, C. (1993) Teoría de la decisión multicriterio: Conceptos, técnicas y aplicaciones. Alianza Editorial. ISBN: 84-206-8144-X

17.Watson I. (1997). Applying Case-Based Reasoning: Techniques for Enterprise Systems. Morgan Kaufmann.

18.Watson, I. and Marir, F. (1994). Case-Based Reasoning: A Review. The Knowledge Engineering Review. Vol. 9. No. 4. pp. 355-381. 